\title{
Cross-Sectional Questionnaire Study on PMS/PMDD and Stress Factors in the Life of Female Medical Students
}

\author{
Jinko Yokota ${ }^{1}$, Michiko Nohara ${ }^{2}$, Toshiko Kamo ${ }^{3}$, Fumi Horiguchi" and Keiko Uchida \\ ${ }^{1}$ Health Care Center, Tokyo Women's Medical University, Japan \\ ${ }^{2}$ Department of Food and Nutrition, Tokyo Kasei University, Japan \\ ${ }^{3}$ Wakamatsu-Cho Mental and Skin Clinic, Japan \\ ${ }^{4}$ The Fumi Horiguchi Institute for Research and Education in Women's Health, Japan
}

*Corresponding author: Jinko Yokota, Health Care Center, Tokyo Women's Medical University, Japan

\begin{abstract}
Purpose: To investigate relationship among PMS/PMDD and stress factors, menstrual distress, psychological distress, menstrual cycle and life habits in female medical student's life.
\end{abstract}

Method: The authors conducted a survey among all students of a medical university in Tokyo between October 15 and 27, 2015, using anonymous self-administered questionnaires. The questionnaire assessed cognitions about 18 stress factors in student life, the premenstrual symptoms screening tool (PSST), menstrual-associated symptoms (modified Menstrual Distress Questionnaire: mMDQ), psychological distress (the Kessler 6), menstrual cycle, age, grade, height, weight, smoking and drinking habits, exercise habits, and eating habits.

Results: We had 56 (13.6\%) moderate to severe PMS, and $10(2.4 \%)$ participants were considered PMDD cases in 412 responded. Among PMS group (moderate to severe PMS/ PMDD) there were compared with the health group (No/mild PMS) significantly many who felt stressed by interpersonal relationships, changes in climate and physical condition, menstruation and physique. In the MDQ, too, complaints about pain, difficulties in concentrating, behavioral changes, autonomic reaction, water retention, and the tendency toward depression pronounced. We could not detect any direct relationships between abnormal menstrual cycles and life habits (smoking, drinking, exercise, and eating).

Conclusion: Approximately $16 \%$ of our female medical students suffer from moderate to severe PMS/PMDD and constantly perceive not only interpersonal relations but also their own physical condition and body shape as excessively stressful. For us, the medical staff, the PMS such as public health issue in this field is of paramount importance, and is felt urgent by female doctors.

\section{Keywords}

Premenstrual syndrome, Stress factor, Medical student, Life habits, Menstrual distress, Psychological distress

\section{Introduction}

Menstruation-related ailments such as premenstrual syndrome (PMS), premenstrual dysphoric disorder, and dysmenorrhea can abstract the health of women of reproductive age [1]. Severe PMS and PMDD was not only high risk factor of developing perimenoposal depression and postnatal depression, but anxiety disorder and panic disorder in woman's life stage [1]. In addition, PMD/PMDD symptoms are more common and more severe in high-revel educated women in different countries [2]. On the other hand, stress and traumatic events are possible risk factors for PMS [1]. It is urgent issue for female medical students to prevent developing psychological distress such as depression and anxiety disorder and cope with PMS/PMDD symptoms.

Previous our study was reported that the prevalence of PMS/PMDD in female medical students was higher than non-medical students [3]. We suspected that the reason why the higher prevalence of PMS/PMDD was influenced by stressful students life due to busy curriculum in the medical university and higher education [3]. But there were few studies for the major stress factor for female students' life in medical college.

Citation: Yokota J, Nohara M, Kamo T, Horiguchi F, Uchida K (2021) Cross-Sectional Questionnaire Study on PMS/PMDD and Stress Factors in the Life of Female Medical Students. Int J Womens Health Wellness 7:128. doi.org/10.23937/2474-1353/1510128

Accepted: November 29, 2021: Published: December 01, 2021

Copyright: (C) 2021 Yokota J, et al. This is an open-access article distributed under the terms of the Creative Commons Attribution License, which permits unrestricted use, distribution, and reproduction in any medium, provided the original author and source are credited. 
Also there was a new question why students prefer to take pain-killer for the self-care of their pre and duringmenstrual symptoms. We suggested that it is overlapped the symptoms of the menstruation-related ailments such as among PMS/PMDD and dysmenorrhea.

The purpose of this study was to clarified relationship among PMS/PMDD and stress factors, menstrual distress, psychological distress, menstrual cycle and life habits in female medical student's life.

\section{Method}

\section{Sample and data collection}

The authors conducted a survey among all students of a medical university in Tokyo between October 15 and 27, 2015, using anonymous self-administered questionnaires. The survey was carried out by distributing questionnaires within the lecture rooms before or after morning lectures for each school year. The completed questionnaires were collected directly from the lecture rooms during the day.

\section{Measures}

The questionnaire assessed cognitions about 18 stress factors in student life, the premenstrual symptoms screening tool (PSST), menstrual-associated symptoms (modified Menstrual Distress Questionnaire), psychological distress (the Kessler 6), menstrual cycle, age, grade, height, weight, smoking and drinking habits, exercise habits, and eating habits [4-6].

We measured PMS and PMDD by using the premenstrual symptoms screening tool (PSST) [4]. The criteria for PMDD: At least one of the four core symptoms (irritability, dysphoria, tension, lability of mood) as severe and at least 4 additional symptoms as moderate to severe. The symptoms interfered severely with their ability to function in at least one of five domains (work efficiency/ productivity, social life, home responsibilities, relationship at work, or relationships at home). The criteria for Moderate to Severe PMS: At least one of the four core symptoms (irritability, dysphoria, tension, lability of mood) as moderate to severe and at least 4 additional symptoms as moderate to severe. The symptoms interfered moderate to severely with their ability to function in at least one of five domains (work efficiency/productivity, social life, home responsibilities, relationship at work, or relationships at home).

Menstrual symptoms were assessed using a modified version of the Menstrual Distress Questionnaire (MDQ) which was developed by Moos [5,7]. A modified version (mMDQ) was created by Akiyama, et al. as the Japanese version of the scale [8]. In Moos' original questionnaire there were 46 items reflecting menstrual complaints, which were classified into 8 subscales via a factor analysis: "pain" (6 items), "impaired concentration" (8 items), "behavior change" (5 items), "autonomic reactions" (4 items), "water retention" (4 items), "negative affect" (8 items), "arousal" (5 items), and "control" (5 items). Because of Japanese culture which is not apply sufficiently the arousal and control subscales by considering Akiyama, et al. the mMDQ comprises only 6 subscales with a total 35 items. Each item is rated on a 6-point scale ranging from 1 ("no symptoms") to 6 ("very strong"). The total score of each subscale was the sum of the item scores, with higher scores meaning more severe symptoms.

The Kessler 6 scale (K6), developed by Kessler, et al. was used to measure psychological distress in the last 30 days [6]. Furukawa, et al. created the Japanese version of the $\mathrm{K} 6$ and confirmed its each reliability and validity [9]. This scale comprises 6 items, with each item being rated on a 5 -point scale ranging from 0 ("none of the time") to 4 ("all of the time"). The total score ranges from 0 to 24 points, with over 13 points being proposed as the cut-off point for a suspected severe mental disorder and 5 points as the cut-off for mild psychological distress in the general population.

Stress factors were assessed using 18 items considered to be sources of stress in student life. These items included lectures, practical training, tutorials, homework, examinations, club activities, part-time job, relationships with others (friends, seniors/juniors, family, and lovers), residence, commuting to school, climate (e.g., excessive heat or cold), physical condition, menstruation, and physique. Each item was rated on a 4-point scale ranging from 0 ("not a problem") to 3 ("a large problem").

\section{Statistical analysis}

All statistical analyses were conducted with SAS version 9.4 (SAS institute Inc., Cary, NC). The results are presented as means and standard deviations or as frequency distributions. We used variance (ANOVA), the Kruskal-Wallis $\mathrm{H}$-test, and the chi-square test to determine differences in study variables between PMDD/severe to moderate PMS group and mild to non PMS group. A.P $<0.01$ was considered to indicate a statistically significant difference.

\section{Ethical considerations}

The research purpose, the voluntariness of participation, the measured taken to protect participants' personal information, and how the data would be handled was indicated on the cover page of the questionnaire. Completing and handing in the questionnaire was regarded as giving consent to participate. The present study was conducted with the approval of the Tokyo Women's Medical University Ethics Committee (Approval number 3612-R).

\section{Result}

The questionnaire was distributed to 602 students, 
Table 1: Subject characteristics.

\begin{tabular}{|l|l|}
\hline Variable & Subjects (n = 412) \\
\hline Age, y & $21.7 \pm 2.3$ \\
\hline Height, cm & $158.8 \pm 5.2$ \\
\hline Weight, kg & $49.8 \pm 5.9$ \\
\hline Body mass index, kg/m² & $19.7 \pm 1.9$ \\
\hline Smoking status, (\%) & \\
\hline Never smoked & $389(94.4)$ \\
\hline Former smoker & $7(1.7)$ \\
\hline Current smoker (<20 cigarettes per day) & $3(0.7)$ \\
\hline Current Smoker (> 20 cigarettes per day) & $1(0.2)$ \\
\hline No answer & $12(2.9)$ \\
\hline Alcohol consumption, (\%) & \\
\hline None & $96(23.3)$ \\
\hline A few times a month & $198(48.1)$ \\
\hline A few times a week & $104(25.2)$ \\
\hline Almost every day & $2(0.5)$ \\
\hline No answer & $12(2.9)$ \\
\hline Exercise (\%) & $114(27.7)$ \\
\hline Almost every day & $26(6.3)$ \\
\hline Few times a week & $13(3.2)$ \\
\hline Few times a month & $176(42.7)$ \\
\hline None & $11(27.9)$ \\
\hline No answer & \\
\hline Dietary habit (\%) & \\
\hline Almost regularly & $17.2)$ \\
\hline Sometimes irregularly & $162.9)$ \\
\hline Almost irregularly & 15 (1) \\
\hline No answer & \\
\hline
\end{tabular}

of which 412 responded (68.4\%) in total (across all grades). Participants mean age was $21.7 \pm 2.3$ years, their mean height was $158.8 \pm 5.2 \mathrm{~cm}$. their mean weight was $49.8 \pm 5.9 \mathrm{~kg}$ and mean body mass index was $19.7 \pm 1.9$. Table 1 shows the characteristics of all study subjects. By year, 94 participants $(22.8 \%)$ were in their first year, $70(17.0 \%)$ in their second year, 67 $(16.3 \%)$ in their third year, $76(18.4 \%)$ inn their fourth year, $51(12.4 \%)$ in their fifth year, and 54 (13.1\%) in their sixth year. Three hundred eighty-nine (94.4\%) participants were non-smokers either did not drink alcohol or drank very little. Two hundred an fifteen (52.2\%) participants exercised more than a few times a week, and 259 (62.9\%) ate three meals almost daily. Forty-nine $(11.9 \%)$ of participants reported always having an irregular menstrual cycle, and $173(42.0 \%)$ of participants reported having a sometime irregular cycle.

Table 2 shows the means and standard deviations for the 18 stress factors. Each item was rated on a 4-point scale ranging from 0 ("not a problem") to 3 ("a large
Table 2: Means scores for severity of stress factors.

\begin{tabular}{|l|l|}
\hline Variable & Mean \pm SD \\
\hline Examinations & $2.6 \pm 0.7$ \\
\hline Practice & $1.7 \pm 0.8$ \\
\hline Menstruation & $1.6 \pm 0.9$ \\
\hline Lectures & $1.5 \pm 0.8$ \\
\hline Club Activities & $1.5 \pm 0.9$ \\
\hline Physique & $1.5 \pm 0.9$ \\
\hline Commuting to school & $1.4 \pm 1.0$ \\
\hline Physical condition & $1.4 \pm 0.9$ \\
\hline Tutorials & $1.3 \pm 0.8$ \\
\hline $\begin{array}{l}\text { Relationship (upperclassmen and } \\
\text { underclassmen) }\end{array}$ & $1.3 \pm 0.9$ \\
\hline Climate & $1.3 \pm 0.9$ \\
\hline Homework & $1.2 \pm 0.9$ \\
\hline Relationship (friends) & $1.2 \pm 0.8$ \\
\hline Part-time job & $1.1 \pm 1.0$ \\
\hline Relationship (lover) & $1.0 \pm 0.9$ \\
\hline Relationship (family) & $1.0 \pm 0.9$ \\
\hline Residance & $0.9 \pm 0.9$ \\
\hline Extramural activities & $0.8 \pm 0.8$ \\
\hline
\end{tabular}

problem"). The factors perceived as causing the greatest stress were examinations, training, menstruation, lecture, club activities, and physique. Table 3 shows the prevalence of PMDD, moderate to severe PMS. In the total number of participants, $10(2.4 \%)$ of participants met the diagnostic criteria for PMDD and 56 (13.6\%) of participants met the diagnostic criteria for moderate to severe PMS.

Figure 1 show differences in stress factors among severe to moderate PMS/PMDD (PMS group) and No/ mild PMS (health group). Each bar in the Figure 1 is the percentage of participants who answered "a large problem" and "a little problem" in each student life. Among PMS group there were compared with the health group significantly many who felt stressed interpersonal relationships, changes in climate and physical condition, menstruation and physique. Table 4 shows differences in menstrual symptoms types and psychological distress among PMDD, severe to moderate PMS and no/mild PMS. In the MDQ, too, complaints about pain, difficulties in concentrating, behavioral changes, autonomic reaction, water retention, and the tendency toward depression pronounced. We could not detect any direct relationships between abnormal menstrual cycles and life habits (smoking, drinking, exercise, eating).

\section{Discussion}

We conducted a survey among all students of one medical school to determine the prevalence of PMS/ PMDD, menstrual cycle, menstrual symptoms, stress factors, and psychological distress among female medical students. 
Table 3: Prevalence of PMS/PMDD among medical students.

\begin{tabular}{|l|l|l|l|}
\hline & PMDD & Moderate to Severe PMS & No/Mild PMS \\
\hline Number of students (\%) & $10(2.4 \%)$ & $56(13.6 \%)$ & $346(84.0 \%)$ \\
\hline
\end{tabular}

Table 4: Differences in menstrual symptoms types and psychological distress among PMDD, Severe to Moderate PMS, and No/ Mild PMS.

\begin{tabular}{|l|l|l|l|l|}
\hline & PMDD & Moderate to Severe PMS & No/Mild PMS & P-value \\
\hline MDQ & & & & \\
\hline Pain & $18.6(4.4)$ & $19.6(6.9)$ & $14.1(5.6)$ & $<0.001$ \\
\hline Concentration & $21.7(7.4)$ & $18.1(8.1)$ & $12.1(4.7)$ & $<0.001$ \\
\hline Behavioral Change & $21.2(5.6)$ & $16.6(5.4)$ & $10.5(5.0)$ & $<0.001$ \\
\hline Autonomic reaction & $12.8(5.8)$ & $8.2(4.3)$ & $5.8(2.8)$ & $<0.001$ \\
\hline Water retention & $12.7(5.8)$ & $11.1(4.3)$ & $7.9(3.5)$ & $<0.001$ \\
\hline Negative affect & $32.3(5.7)$ & $25.4(10.1)$ & $13.5(6.6)$ & $<0.001$ \\
\hline K6 & & & & $<0.001$ \\
\hline$<5$ & $1(10.0 \%)$ & $19(33.9 \%)$ & $78(22.5 \%)$ & $2(0.6 \%)$ \\
\hline$>5$ & $9(90.0 \%)$ & $36(64.3 \%)$ & $1(1.8 \%)$ & $26.9 \%)$ \\
\hline No answer & & & \\
\hline
\end{tabular}

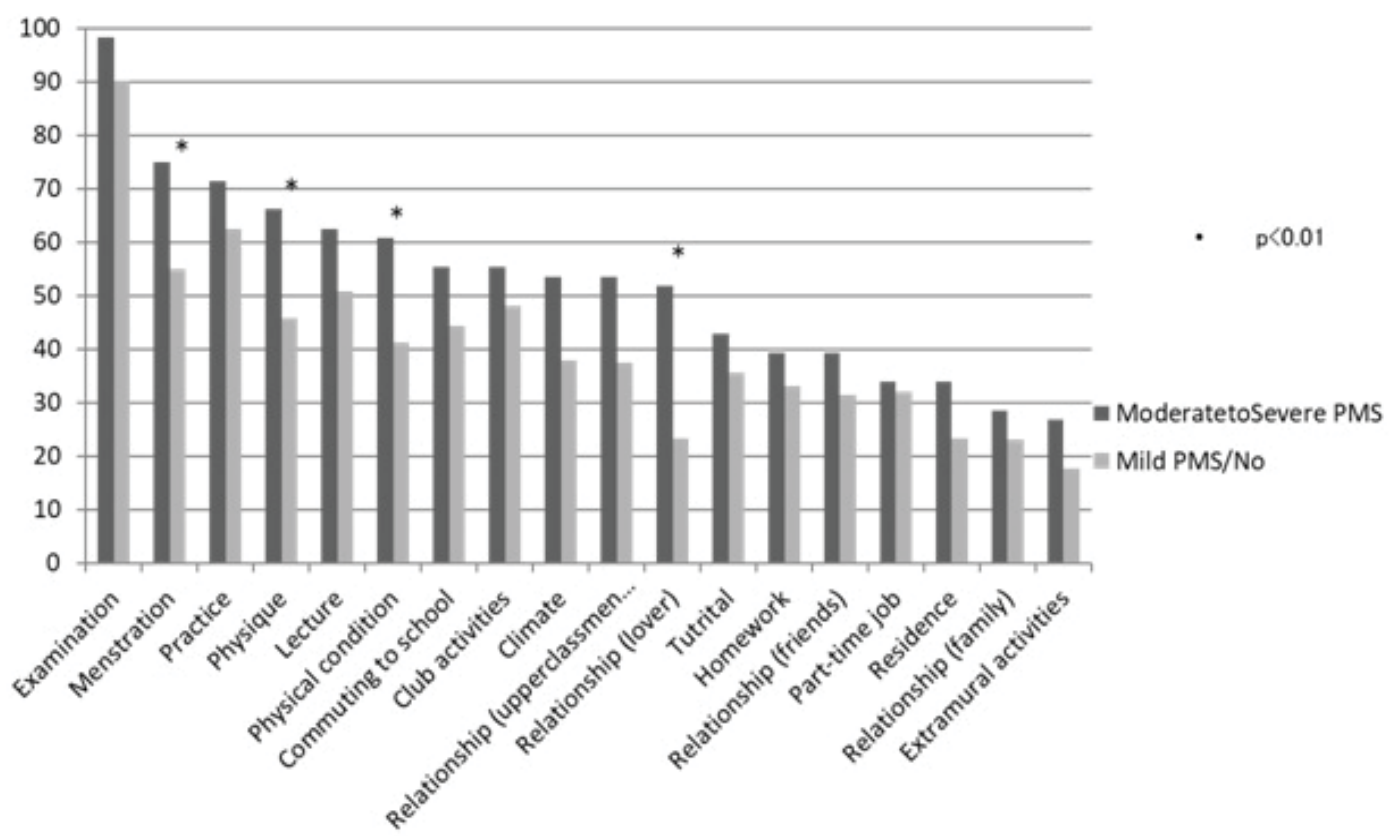

Figure 1: Differences in stress factors among Severe to Moderate PMS, and NO/Mild PMS.

Thirteen point three percentages of participants of this survey suffered from moderate to severe PMS, 2.4\%for PMDD. With regard to frequency of moderate to severe PMS and PMDD in Japan, Takeda, et al. on senior high school students, a percentage of $11.8 \%$ was reported for PMS and 2.6\%, for PMDD [10]. Yonkers, et al. reported in their seminar the prevalence of moderate to severe PMS in retrospective and prospective studies was $5-8 \%$ of women with hormonal cycles [1]. The prevalence of PMS/PMDD in our data was similar to Takeda's study in young women in Japan, and higher than Yonkers' report in the women with hormonal cycle.

In a comparison between Moderate to Severe PMS afflicted students and No/Mild PMS we considered the Moderate to Severe PMS group as suffering more from stress than the No/Mild PMS group. Given this result, we could easily imagine that stronger the PMS symptoms are easier one in prone to experience stress in daily life. Sakuma, et al. reported that less stress involved in low menstrual rerated symptom in nursing students, also the student of suffering PMS more easily felt stress in their life [11]. Akimoto, et al. studied the participants of diagnosed PMS/PMDD had a tendency in their negative cognition in re-interpretation for stress coping pattern [12]. Then, the cognitive-behavior therapy might be useful for the participants of PMS/PMDD [1]. 
As stress factors in moderate to severe PMS afflicted students examinations scored first, but menstruation connected problems came in on the second place. As far as menstruation are concerned we can guess that the participants with PMS are perceiving menstruation as stressful because of diminished or vanished symptoms at the beginning of menstruation due to appearance of mental and physical symptoms in the lutal phase before menstruation [1]. On the other hand, examination has to be taken from all medical students whether they are PMS-afflicted or not, are source of stress for everybody.

There was a statistically significant difference in stress factors between moderate to severe PMS group and No/Mild PMS group as far as relationships (lover), physical condition, menstruation and body type were concerned. From this result, we could suppose that PMS students would feel stress at the beginning of the PMS symptoms with menstruation, It can be assumed that students become very sensitive to changes in the physique parallel to PMS symptoms and altered physical constitutions due to hormonal fluctuations which are thought to cause PMS in the first place, and premenstrual symptoms influence even things like relationships (lover).

There was a statistically significant difference in relationship in menstrual distress among PMDD, moderate to severe PMS, and no/mild PMS. Pinar, et al. reported PMS has significantly high in students who have menstrual irregularity, who have dysmenorrhea, who smoke and drink alcohol [13]. Akimoto, et al. reported statistically significant difference in the relationship among PMS/PMDD patient and dysmenorrheal [12]. Our result supports the previous reports.

In terms of relationships between PMS/PMDD and psychological distress, it is important to prevent developing mental illness such as depression which is risk factor for suicide in medical students. Also PMS related to high suicide and accident rates. Tenkir, et al. mentioned that because of severe premenstrual symptoms PMS participants missed classes and missed examination or scored a lower grade [14]. In our study, there is a statistically significant difference among PMDD, moderate to severe PMS, and no/mild PMS in $\mathrm{K} 6$.

In the future we have to put more effort into education about and support of PMS in the way of promoting a deeper understanding of the affliction, not only in auto-coping but also in society at large.

This study has several limitations. First, because of a survey of only one medical university in Tokyo it cannot be considered representative of all female medical students in Japan. Second, it was cross-sectional in nature, which makes it impossible to account for influences of season and time. Third, it was crosssectional study, which makes it is impossible to clarify the causal relationship between stress and PMS symptoms.
Therefore it would be necessary to expand the range of study, use a longitudinal design and perform more detailed analysis such as qualitative research.

\section{Conclusion}

Approximately $16 \%$ of our female medical students suffer from PMS/PMDD and constantly perceive not only interpersonal relations but also their own physical condition and body shape as excessively stressful. For us, the medical staff, the PMS public health issue in this field is of paramount importance, and is felt urgent by female doctors.

\section{References}

1. Yonkers KM, O’Brien PMS, Eriksson E (2008) Premenstrual syndrome. Lancet 371: 1200-1210.

2. Marvan ML, Díaz-Erosa M, Montesinos A (1998) Premenstrual symptoms in mexican women with different education levels. J Psychol 132: 517-526.

3. Yokota J, Shinozaki A, Kamo T, Horiguchi F, Uchida K (2017) A questionnaire study on prevalence of premenstrual syndrome, premenstrual dysphoric disorder, and coping mechanisms among female medical students. J TWMU 1: 1-7.

4. Steiner M, Macdougall M, Brown E (2003) The premenstrual symptoms screening tool (PSST) for clinicians. Arch Women's Ment Health 6: 203-209.

5. Moos RH (1968) The development of Menstrual Distress Questionnaire. Psychosomatic Med 3: 853-867.

6. Kessler RC, Andrews G, Colpe LJ, Hiripi E, Mroczek DK, et al. (2002) Short screening scales to monitor population prevalences and trend in non-specific psychological distress. Psychol Med 32: 959-976.

7. Goto Y, Okuda H (2005) Premenstrual symptoms and their management: Assessment with Menstrual Distress Questionnaire. Bull Fac Health Sci Okayama Univ Med Sch 16: 21-30.

8. Akiyama A, Kayashima K (1979) Studies on the influence of the menstrual cycle upon the physical and mental conditions by means of MDT (Mirror Drawing Test) method. J Jpn Soc Nurs Res 192: 61-66.

9. Furukawa TA, Kawakami N, Saitoh M, Ono Y, Nakane Y, et al. (2008) The performance of the Japanese version of the $\mathrm{K} 6$ and $\mathrm{K} 10$ in the World Mental Health Survey Japan. Int J Methods Psychiatr Res 17: 152-158.

10. Takeda T, Koga S, Yaegashi N (2010) Prevalence of premenstrual syndrome and premenstrual dysphoric disorder in Japanese high school students. Arch Womens Ment Health 13: 535-537.

11. Sakuma $Y$, Kanoya $Y$, Ishimitsu F, et al. (2008) The factors affecting prementrual and menstrual symptoms among young women : Comparison of the lifestyle/health behaivior in nursing students and non-medical vocational school students. J Jpn Soc Nurs Res 31: 25-36.

12. Akimoto Y, Miyaoka Y, Kamo T (2009) Clinical charactaristics and stress coping behaiviors in women with premenstrual symdorome or premenstrual dysphoric disorder. Atomi Gakuen Joshi Gaigaku Bugakubu Kiyou 43: 45-60.

13. Pinar G, Colak M, Oksuz E (2011) Premenstrual Syndrome in Turkish college students and its effects on life quality. Sexual \& Reproductive Healthcare 2: 21-27.

14. Tenkir A, Fisseha N, Ayele B (2002) Prementrual syndrome: Prevalence and effect on academic and social performances of students in Jimma University, Ethiopia. Ethiop. J Health Dev 17: 181-188. 